\title{
Editorial del número especial: El Aprendizaje-Servicio en el Entorno de las Tecnologías Digitales
}

\author{
Editorial of the special issue: Service-Learning in Digital Technology Environments
}

\author{
(iD) Berta Paz-Lourido; bpaz@uib.es \\ Bárbara de-Benito Crosetti; barbara.debenito@uib.es \\ Universitat de les Illes Balears (España)
}

\begin{abstract}
En apenas unas décadas, el Aprendizaje-Servicio (ApS) ha pasado de ser un enfoque pedagógico prácticamente desconocido a convertirse en una de las estrategias educativas de mayor impacto, no sólo en términos de aprendizaje curricular y éxito académico, sino también por su vertiente de servicio solidario a la comunidad (Tapia, 2010). Aprender haciendo no es completamente novedoso. Podemos remontarnos en la historia a múltiples prácticas pedagógicas donde una mirada amplia y no restringida puede identificar huellas del aprendizaje y del servicio (Puig et al., 2011). Lo que sí es genuinamente nuevo es una planificación equilibrada de los proyectos ApS cada vez más exquisita en sus detalles, una búsqueda de significados más allá de la experiencia aislada y al tiempo, un interés en que gocen del soporte adecuado para superar la excepcionalidad de lo puntual en el marco de una entidad educativa comprometida (Bringle y Hatcher, 2000; Furco, 2010; Paz-Lourido et al., 2018); es decir, la institucionalización del ApS.
\end{abstract}

Con ello como meta, son necesarias una serie de políticas, estrategias y organización de prácticas educativas que favorezcan la institucionalización del ApS entendiendo su diversidad de enfoques en el marco internacional (Mejis et al., 2019). La identificación de experiencias en redes nacionales como la Red Española de Aprendizaje-Servicio o internacionales como el Centro Latinoamericano de Aprendizaje y Servicio Solidario ayudan a visibilizar el ApS y se suman a los mapeos sistemáticos como el realizado por el European Observatory of ServiceLearning in Higher Education. La investigación y su difusión en publicaciones científicas como ésta nos permite abrir senderos por los que otros equipos docentes, investigadores y comunitarios podrán seguir avanzando. Con los frutos de éstos y otros estudios madura la propia conceptualización del ApS porque que se construyen nuevos pilares basados en los resultados de su aplicación contextualizada.

La tecnología educativa ha venido para quedarse en el espacio del ApS, pero no sólo para mediar en los procesos (Sparkman, 2020), sino para dotar de nuevos significados aquello que pensábamos relegado al espacio presencial y local, haciendo necesario un marco general que integre las distintas aproximaciones (Faulconer, 2020). De esta manera, dedicar este número especial al ApS nos permite mostrar las múltiples posibilidades de la tecnología para desarrollar, fomentar o potenciar proyectos de ApS orientados a dar respuesta a necesidades reales de nuestro entorno más cercano, pero también de contextos más alejados, configurando proyectos interregionales o incluso transnacionales (Salam, 2019; Marcus et al. 2020). Analizar 
los componentes de experiencias innovadoras facilita su transferencia a entornos similares, visibilizando particularmente el rol que la tecnología puede tener en el desarrollo de estos proyectos (Tapia- Sasot, 2021).

Pero antes de seguir, retomemos los aspectos básicos del ApS: es un enfoque pedagógico, una metodología docente experiencial y una estrategia de compromiso solidario que permite al alumnado trabajar en necesidades reales del entorno con la finalidad de mejorarlo. Los proyectos de ApS son proyectos equilibrados donde el aprendizaje y el servicio tienen ambos el mismo peso y se articulan para crear impactos en todos los agentes implicados. Tal y como reconoció la Conferencia de Rectores de las Universidades Españolas en su conocido documento técnico Institucionalización del Aprendizaje-Servicio como estrategia docente dentro del marco de la responsabilidad Social Universitaria para la promoción de la Sostenibilidad en la Universidad de 2015, el ApS puede desarrollarse en todos los niveles educativos, desde muy diversas áreas académicas y en colaboración participativa con entidades como asociaciones, ONGs, fundaciones, entidades públicas, entre otras; encaminadas a conseguir una sociedad más justa, inclusiva, saludable y sostenible. Además, las acciones de servicio pueden orientarse a las diferentes dimensiones de la sostenibilidad desde ámbitos como el medio ambiente, el patrimonio social, cultural y artístico, el apoyo a la educación, la atención a personas vulnerables, la inclusión, la promoción de la salud, el fomento de la justicia social, entre muchas otros (CRUE, 2015).

En este marco de las múltiples posibilidades del ApS, los Objetivos de Desarrollo Sostenible suponen vías amplias en los que enmarcar estos proyectos que promueven la adquisición de competencias mediante el fomento del espíritu crítico y el compromiso social en la resolución creativa de problemas (Batlle, 2020). Pero, además, dirigiendo las acciones de servicio a necesidades concretas se contribuye a dar respuesta a los retos globales que vivimos en la actualidad. Es por ello que, en línea con Turnley (2007) y Sandia-Saldivia y Montilva-Calderón (2020) en el ApS virtual sigue siendo crucial no sólo "el qué" si no "el cómo", es decir, cómo de participativos han sido los proyectos, qué valores y reflexividad crítica se estimula, cómo las necesidades son planteadas y resueltas, etc., y no únicamente qué tecnologías son utilizadas.

Durante la pandemia COVID-19, un gran número de proyectos de ApS presenciales tuvieron que suspenderse. Sin embargo, esta situación excepcional también ocasionó que otros muchos pudieran continuar gracias a los entornos tecnológicos, sumándose así a aquellos surgidos a la luz de una crisis socio-sanitaria compleja y cambiante (Stefianiak, 2020). Unos y otros han puesto el foco en las recientes necesidades surgidas, pero también en aquellas que ya existían y que la pandemia simplemente hizo más visibles. En cualquier caso, estas propuestas no están exentas de retos, entre ellos, la accesibilidad a la tecnología y la propia formación del profesorado (Hollander et al, 2020).

El ApS en el entorno de las tecnologías digitales ha suscitado un enorme interés en el ámbito académico que se ha visto traducido en un numeroso grupo de artículos adecuados para formar parte de este número especial. Finalmente, forman parte del mismo un total de 13 artículos que representan distintos enfoques, niveles educativos y tipos de estudios, a fin de ilustrar algunos de los aspectos de debate a nivel internacional en esta temática. En cuanto a la institucionalización del ApS en el ámbito universitario, destacamos el trabajo de Juan García, Alberto Izquierdo-Montero y Marta Ruíz-Corbella, titulado El aprendizaje-servicio virtual: una propuesta innovadora desde su institucionalización en la Universidad Nacional de Educación a 
Distancia (UNED), que presenta la experiencia de esta universidad en el diseño, implementación y desarrollo del ApS virtual enmarcada en su proceso de institucionalización.

Continuando con los proyectos desarrollados en educación superior destacamos el trabajo firmado por María Santágueda, María Ángeles LLopis y Francesc Marc Esteve, titulado Una aplicación móvil para trabajar en el Aprendizaje Servicio universitario: usabilidad, adecuación y percepciones de utilidad, en el que recogen las valoraciones de profesorado y estudiantes de distintas titulaciones universitarias de educación, sobre una aplicación desarrollada para dar soporte tecnológico a proyectos de ApS.

En cuanto a proyectos ApS en el marco universitario contamos con varias propuestas, que dan cuenta de la cantidad de experiencias que se están realizando en este nivel educativo. Por un lado, el trabajo de Alexandre Sotelino-Losada y Millán Brea-Castro, incide en el uso de la red social "Instagram" en su estudio titulado Aprendizaje-Servicio Virtual y Pedagogía Social. El proyecto ProAcción Social, una exitosa propuesta desarrollada con estudiantes del Grado de Educación Social, que ayuda a visibilizar socialmente el perfil profesional de esta disciplina académica.

Destacan, especialmente, las experiencias en la formación inicial de profesorado, que consideramos clave para que implementen esa metodología en su desarrollo profesional futuro. En este sentido, varios artículos incorporan el ApS como estrategia educativa dentro de las titulaciones de ciencias de la educación. Entre ellos el firmado por José Luis Lázaro, Mònica Sanromà, Tania Molero e Ivan Sanz, y que lleva por título La formación en competencias digitales de los futuros docentes: una experiencia de Aprendizaje-Servicio en la universidad, en el que presentan los resultados de la implementación de ApS durante un período de siete años, con estudiantes del doble grado de Educación Infantil y Primaria, juntamente con el Grado de Pedagogía y que han dado respuesta a necesidades planteadas por los centros escolares, elaborando materiales educativos personalizados de forma colaborativa. O el caso presentado por Domingo Mayor, con el trabajo titulado Aprendiendo a cuidar-nos, una experiencia de aprendizaje-servicio virtual en educación infantil, en el que nos muestra las posibilidades que ofrece el ApS mediado por tecnologías de comunicación digital, no sólo para la mejora de la formación académica sino también para establecer vías de colaboración participativa, entre distintos niveles educativos, para un aprendizaje recíproco. Por su parte, Isabel Dans y Cristina Varela en su artículo Digitalización, compromiso y resiliencia. Proyecto de aprendizaje -servicio con futuros docentes, se centran en las competencias digitales docentes de futuros maestros y maestras de educación primaria, ofreciendo un servicio dirigido a la población infantil vulnerable. El artículo firmado por Carlos Corrales, Johanna Herrera y Patricia Erazo donde nos detallan el proyecto Aprendizaje-Servicio Virtual en tiempos de emergencia sanitaria: una experiencia de refuerzo académico de docentes en formación. Este ApS fue desarrollado en Ecuador y estuvo dirigido a mitigar la desigualdad producida por la pandemia, mediante acciones de refuerzo académico virtual de estudiantes universitarios a niños de entre 4 y 11 años. Por último, los autores Rakel Gamito, Eider Hermoso, Irati León y Lander Bilbao dirigen la mirada hacia la formación inclusiva y la atención a la diversidad con su propuesta AprendizajeServicio para acercar la robótica educativa a las personas con parálisis cerebral y promover las competencias docentes en estudiantes del Grado de Educación Primaria en colaboración con ASPACE Álava. 
Otras aportaciones de interés se refieren específicamente a la formación en ApS. Así, en primer lugar, encontramos la propuesta de Marta Gómez-Gómez, Inmaculada Gómez-Jarabo y Bienvenida Sánchez, en su trabajo La formación en Aprendizaje-Servicio Solidario ante el reto de los escenarios virtuales de aprendizaje en Educación Superior, donde a partir del trabajo colaborativo entre diferentes universidades, forman en ApS a estudiantes de los Grados de Educación Infantil y Educación Social, apuntando datos relevantes para la transferibilidad de su proyecto. En segundo lugar, y en el contexto académico en Sudáfrica, El alineamiento constructivo de un curso online de aprendizaje-servicio, pone el foco en la formación en ApS mediante entornos virtuales, incidiendo Joana Bezerra y Sharli Paphitis en la manera en que aquello que es característico del ApS debe también impregnar la formación sobre este tipo de metodología didáctica.

Continuando con otras experiencias del ámbito universitario, en el artículo Aprendizaje-servicio para la formación en atención farmacéutica, enfrentando los retos de la pandemia COVID-19, Vanesa Carmona, Miguel Vázquez-Velasco, Carmen del Campo y Giorgio Giorgi, nos proponen un proyecto desarrollado con estudiantes del Grado de Farmacia donde, a través del asesoramiento farmacéutico contribuyen a fomentar la equidad en la en salud de colectivos vulnerables como el LGTBI+. En cuanto a las perspectivas y las valoraciones del colectivo de estudiantes universitarios sobre el ApS virtual, Irene Culcasi, Luciano Romano y Maria Cinque, nos presentan un estudio titulado Aprendizaje-Servicio Virtual 100\% online: un estudio de caso en una muestra de estudiantes universitarios italianos, que incluye a alumnado de distintas titulaciones de una universidad italiana en el que desarrollaron proyectos ApS totalmente mediados por tecnología y cuyos resultados aportan indicaciones para la práctica docente y futuras líneas de investigación.

Finalmente, forma parte de este número especial, el trabajo Aprendizaje socioemocional en la comunicación online a través de la metodología de Aprendizaje-Servicio en adolescentes", en el que Ana Cebollero nos muestra una propuesta donde el alumnado de cuarto de educación secundaria da respuesta a necesidades que presentan estudiantes de segundo curso, también de secundaria, ilustrando las posibilidades que el ApS tiene incluso dentro del mismo nivel educativo.

Confiamos en que esta selección de artículos goce de su merecido interés y pueda contribuir a la institucionalización del ApS en todos los niveles educativos, pues todavía hoy sigue siendo una prioridad. En el ámbito universitario español, la reciente Declaración de Canarias sobre Aprendizaje-Servicio en la Educación Superior (APS(U), 2021)., ha de suponer un paso más allá de la ya citada recomendación de la CRUE, visibilizando no sólo los proyectos desarrollados sino también el compromiso para su continuidad, mejora y transferencia. Este documento, que es uno de los frutos del X Congreso Nacional y IV Internacional de Aprendizaje-Servicio celebrado en Las Palmas de Gran Canaria en 2021, supone la cristalización de los deseos de las personas participantes en dicho evento, miembros de la Red y/o la Asociación de Aprendizaje-Servicio Universitario. Desde este editorial queremos sumar nuestro apoyo público a dicha Declaración a fin de animar a que cada vez más universidades se adhieran a la misma. ${ }^{1}$

1 https://www.apsuniversitario.org 


\section{REFERENCIAS}

APS(U) (2021). Declaración de Canarias sobre aprendizaje-servicio en la educación superior. [Declaración]. https://bit.ly/3Aj7hjG

Batlle, R. (2020). Aprendizaje-Servicio. Compromiso social en acción. Santillana.

*Bezerra, J., y Paphitis, S. (2021). El alineamiento constructivo de un curso online de aprendizaje-servicio. Edutec. Revista Electrónica De Tecnología Educativa, (78), 149164. https://doi.org/10.21556/edutec.2021.78.2291.

Bringle, R.G. y Hatcher, J.A. (2000). Institutionalization of service learning in higher education. The journal of higher education, 71 (3), 273-290.

*Carmona Mata, V. (2021). Aprendizaje-servicio para la formación en atención farmacéutica, enfrentando los retos de la pandemia COVID-19. Edutec. Revista Electrónica De Tecnología Educativa, (78), 165-179. https://doi.org/10.21556/edutec.2021.78.2161.

*Cebollero Salinas, A. (2021). Aprendizaje socioemocional en la comunicación online a través de la metodología de Aprendizaje-Servicio en adolescentes . Edutec. Revista Electrónica De Tecnología Educativa, https://doi.org/10.21556/edutec.2021.78.2193.

(78), 196-210.

Comisión de Sostenibilidad - CRUE (2015). Institucionalización del Aprendizaje-Servicio como estrategia docente dentro del marco de la Responsabilidad Social Universitaria para la promoción de la Sostenibilidad en la Universidad. https://bit.ly/3Dg9A8Y

*Corrales Gaitero, C., Herrera Segarra, J., y Erazo Ortega, P. (2021). Aprendizaje-Servicio virtual en tiempos de emergencia sanitaria. Edutec. Revista Electrónica De Tecnología Educativa, (78), 99-113. https://doi.org/10.21556/edutec.2021.78.2153.

*Culcasi, I., Romano, L., y Cinque, M. (2021). Aprendizaje-Servicio Virtual 100\% online. Edutec. Revista Electrónica De Tecnología Educativa, (78), 180-195. https://doi.org/10.21556/edutec.2021.78.2255.

*Dans Álvarez de Sotomayor, I., y Varela Portela, C. (2021). Digitalización, compromiso y resiliencia. Proyecto de aprendizaje -servicio con futuros docentes. Edutec. Revista Electrónica De Tecnología Educativa, (78), 85-98. https://doi.org/10.21556/edutec. 2021.78.2233.

Faulconer, E. (2020). eService-Learning: A Decade of Research in Undergraduate Online Service-learning, American Journal of Distance Education. 35(2), 100117.http://doi.org/10.1080/08923647.2020.1849941.

Furco, A. (2010). The engaged campus: Toward a comprehensive approach to public engagement. British Journal of Educational Studies, 58 (4), 375-390.

*Gamito Gomez, R., Hermoso Larzabal, E., Leon Hernandez, I., y Bilbao Antia, L. (2021). Aprendizaje-Servicio para acercar la robótica educativa a las personas con parálisis 
cerebral y promover las competencias docentes. Edutec. Revista Electrónica De Tecnología Educativa, (78), 114-130. https://doi.org/10.21556/edutec.2021.78.2213.

*García, J., Izquierdo-Montero, A., \& Ruiz-Corbella, M. (2021). El aprendizaje-servicio virtual: una propuesta innovadora desde su institucionalización en la Universidad Nacional de Educación a Distancia (UNED). Edutec. Revista Electrónica De Tecnología Educativa, (78), 8-21. https://doi.org/10.21556/edutec.2021.78.2241.

*Gómez-Gómez, M., Gómez-Jarabo, I., y Sánchez Alba, B. (2021). La formación en Aprendizaje Servicio Solidario ante el reto de los escenarios virtuales de aprendizaje en Educación Superior. Edutec. Revista Electrónica De Tecnología Educativa, (78), 131-148. https://doi.org/10.21556/edutec.2021.78.2257.

Hollander, A., Vavasseur, C. y Robicheaux, H. (2020). A Service-Learning Approach for Faculty Development Focused on Remote Delivery of Courses During a Pandemic. Journal of Service-Learning in Higher Education, 11(2), 1-13.

*Lázaro-Cantabrana, J. L., Sanromà Giménez, M., Molero Aranda, T., y Sanz Benito, I. (2021). La formación en competencias digitales de los futuros docentes: una experiencia de Aprendizaje-Servicio en la universidad. Edutec. Revista Electrónica De Tecnología Educativa, (78), 54-70. https://doi.org/10.21556/edutec.2021.78.2243.

Marcus, V. B., Atan, N. A., Yosof, S. M., y Tahir, L. (2020). A systematic review of e-service learning in higher education. International Journal of Interactive Mobile Technologies, 14(6), 4-14. https://doi.org/10.3991/ijim.v14i06.13395.

* Mayor Paredes, D. (2021). Aprendiendo a Cuidar-nos, una experiencia de Aprendizaje-Servicio virtual en educación infantil. Edutec. Revista Electrónica De Tecnología Educativa, (78), 71-84. https://doi.org/10.21556/edutec.2021.78.2169.

Meijs, L., Maas, St., y Aramburuzabala, P. (2019). Institutionalization of Service-learning in European Higher Education. In P. Aramburuzabala, L. Mcllrath, y H. Opazo (Eds.). Embedding Service Learning in European Higher Education (213-229). Routledge

Paz-Lourido, B., Negre, F., Verger, S. y de-Benito, B. (2018). El aprendizaje-servicio en la educación superior: de las experiencias puntuales a la institucionalización. En T. Lleixa, B. Gros, T. Mauri, y J.L. Medina (eds.) Educación 2018-2020. Retos, tendencias y compromisos. IRE-Universidad de Barcelona

Puig, J.M., Gijón, M., Martín X., y Rubio., L. (2011). Aprendizaje-servicio y educación para la ciudadanía. Revista de educación, 1, 45-67.

Salam, M., Iskandar, D. N., Ibrahim, D. H., y Farooq, M. (2019) Service learning in higher education: a systematic literature review. Asia Pacific Education Review, 20, 573-593.

Sandia-Saldivia, B., y Montilva-Calderón, J. (2020). Tecnologías Digitales en el AprendizajeServicio para la Formación Ciudadana del Nuevo Milenio. RIED. Revista Iberoamericana de Educación a Distancia, 23(1), 129-148.http://dx.doi.org/10.5944/ried.23.1.24138. 
*Santágueda Villanueva, M., LLopis Nebot, M. Ángeles, y Esteve Mon, F. M. (2021). Una aplicación móvil para trabajar en el aprendizaje servicio universitario: usabilidad, adecuación y percepciones de utilidad. Edutec. Revista Electrónica De Tecnología Educativa, (78), 22-37. https://doi.org/10.21556/edutec.2021.78.2231.

*Sotelino-Losada, A., y Brea Castro, M. (2021). Aprendizaje-Servicio Virtual y Pedagogía Social. El proyecto ProAcción Social. Edutec. Revista Electrónica De Tecnología Educativa, (78), 38-53. https://doi.org/10.21556/edutec.2021.78.2251.

Sparkman, K. N., Vajda, A., y Belcher, T. (2020). Advancing Human Services Education: Exploratory Study of International Service-Learning and Digital Pedagogy. Journal of Service-Learning in Higher Education, 18, 1-18.

Stefaniak, J. A. (2020). Systems View of Supporting the Transfer of Learning through E-ServiceLearning Experiences in Real-World Contexts. Tech Trends, 64, 561-569.

Tapia-Sasot, M. R. (2021). Ventanas abiertas al aprendizaje y servicio solidario virtual: como desarrollar proyectos de aprendizaje-servicio solidario mediados por tecnologías. CLAYSS.

Tapia, N. (2010). Aprendizaje y servicio solidario: en el sistema educativo y las organizaciones juveniles. Ciudad Nueva.

Turnley, M. (2007). Integrating critical approaches to technology and service-learning projects. Technical Communication Quarterly, 16(1), 103-123. https://doi.org/10.1080/10572250709336579.

*Artículos que forman parte del número especial.

\section{Para citar:}

Paz-Lourido, B., y de-Benito, B. (2021). Editorial del número especial: El Aprendizaje-Servicio en el Entorno de las Tecnologías Digitales. Edutec. Revista Electrónica De Tecnología Educativa, (78), 1-7. https://doi.org/10.21556/edutec.2021.78.2317 